Bei den durchschnittlichen motorischen Schwellenwerten und den MEPAmplituden bestanden insgesamt keine signifikanten Unterschiede zwischen den Migränepatienten und den Kontrollprobanden. Dennoch zeigten sich bei den Migränepatienten leichte Schwankungen: Der RMT korrelierte mit der Anzahl der Tage seit dem letzten Migräneanfall - je weiter die Attacke zurücklag desto geringer war die erforderliche muskelkontraktionsauslösende Schwellendosis.
Fazit: Bei Migränepatienten verändert sich die Exzitabilität des motorischen Kortex in zeitlicher Abhängigkeit vom letzten Migräneanfall. Dies deutet auf mögliche plastische Veränderungen im Gehirn der Patienten im Verlauf des Migränezyklus hin.

Dr. Barbara Kreutzkamp

Cortese F et al. Excitablity of the motor cortex in patients with migraine changes with the time elapsed from the last attack. J Headache Pain 2017;18:2

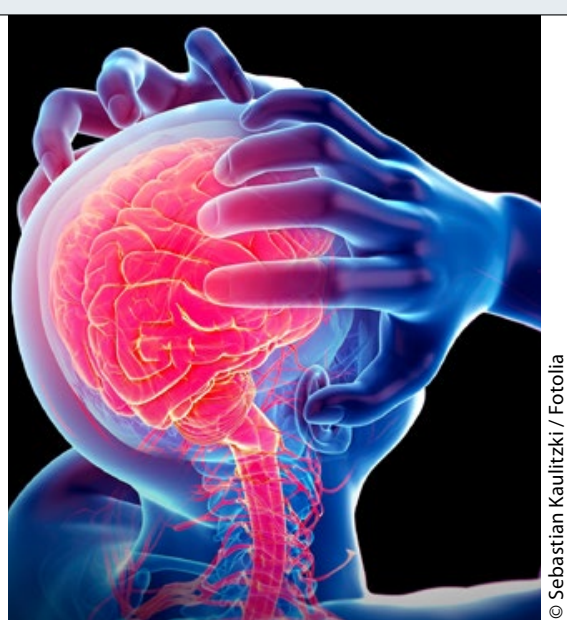

Im Laufe des Migränezyklus verändert sich die Erregbarkeit des Motorkortex.

\section{Fünf entscheidende Merkmale für die Prognose von Rückenschmerzen}

In einem Jahr ist der Schmerz fort - das gilt für die Mehrheit der Patienten mit Kreuzweh. Doch ein Jahr, zumal unter Schmerzen, kann lang sein. Ein Prognoseinstrument für die ersten drei Monate haben australische Mediziner entwickelt.

$R^{2}$ ïckenschmerzen im Lumbalbereich haben üblicherweise eine gute Prognose. Zwar konnten die in manchen Leitlinien angegebenen Erholungsraten von $90 \%$ binnen sechs Wochen in Studien nicht bestätigt werden. Dennoch darf die große Mehrheit der Patienten - etwa $70 \%$ - damit rechnen, in einem Jahr beschwerdefrei zu sein.

Ein australisches Ärzteteam um Tatiane da Silva von der Macquarie University in Sydney hat sich damit beschäftigt herauszufinden, welche Faktoren den Verlauf von akuten Kreuzschmerzen bestimmen. Die dafür analysierten Daten stammten von 1.070 Patienten mit akuten Kreuzschmerzen.
Ziel der Analyse war es vorherzusagen, wie es eine Woche, einen Monat und drei Monate später um die Patienten und ihre Schmerzen bestellt sein würde. Fünf Merkmale, jeweils dreifach gestuft, gaben schließlich den prognostischen Ausschlag: Dauer der aktuellen Schmerzepisode (7-14, 15-23, 24-56 Tage); Zahl vorangegangener Episoden $(0-2,3-8$, 9-150); Depressionsscore (0-3, 4-6, 7-10, je höher, desto depressiver); die Schmerzlinderung nach einer Woche (Verbesserung um 4 Punkte oder mehr, um 2-3 Punkte, um 1 Punkt bzw. gleichbleibend bzw. Verschlechterung auf einer numerischen Rating-Skala); und die Schmerzintensität nach einer Woche (2-

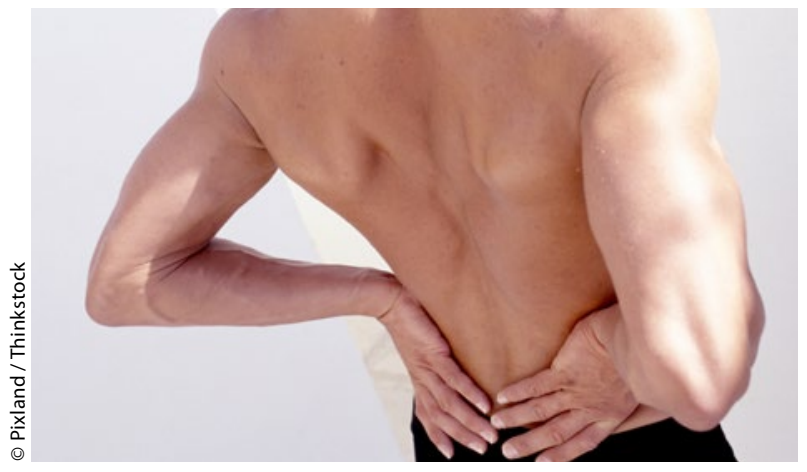

Womöglich können Mediziner bald die Genesungszeit ihrer Rückenschmerzpatienten vorhersagen.
$4,5-7,8-10$ auf einer numerischen Rating-Skala).

Aus diesen Faktoren bildeten da Silva und Kollegen ein Vorhersagewerkzeug, das es ermöglicht, die Wahrscheinlichkeit der Gesundung eine Woche, einen Monat und drei Monate später vorherzusagen. Beispielsweise hätte ein Patient mit einer Dauer der akuten Episode von 7-14 Tagen, 0-2 früheren Episoden, einem Depressionsscore von 0-3, mäßig bis stark gelinderten Schmerzen nach einer Woche, die sich zudem im Bereich leichter Beschwerden befinden, eine Chance von $97 \%$, dass seine Kreuzschmerzen innerhalb von drei Monaten verschwunden sind. Analog, jedoch mit niedrigeren Wahrscheinlichkeitswerten, verläuft die Prognose für die erste Woche und den ersten Monat nach Erhebung der Parameter.

Das Modell erreichte in der C-Statistik einen Wert von 0,76, der für eine gute, wenn auch nicht starke Trennschärfe steht. Angaben zu Spezifität und Sensitivität machten die Forscher nicht, vorhergesagte und beobachtete Befunde stimmten aber recht gut überein. Allerdings betonen da Silva und Mitarbeiter, das Modell müsse extern validiert werden. Zudem sei in einer randomisierten und kontrollierten Studie zu klären, ob sich mit dem Vorhersagewerkzeug die Ergebnisse für die Patienten verbessern und Kosten senken ließen.

Dr. Robert Bublak

Da Silva T et al. Predicting recovery in patients with acute low back pain: A Clinical Prediction Model. Eur J Pain 2017 Jan 20 